\title{
Effects of methotrexate on glycosaminoglycan production by scleroderma fibroblasts in culture
}

Frank $\mathrm{H} J$ van den Hoogen, Peter $M$ van der Kraan, Agnes $M$ T Boerbooms, Wim B van den Berg, Henk J J van Lier, Levinius B A van de Putte

\begin{abstract}
Objective-To determine the effects of increasing concentrations of methotrexate on the proliferation and glycosaminoglycan (GAG) synthesis of cultured dermal fibroblasts from patients with scleroderma.

Methods-Cultured dermal fibroblasts from nine patients with scleroderma and nine normal volunteers were grown for 72 hours in media containing various concentrations of methotrexate. The GAG synthesis in each cell was measured after incubating the fibroblasts with $\left[{ }^{3} \mathrm{H}\right]$ glucosamine and $\left[{ }^{35} S\right]$ sulphate.

Results-A negative correlation was found between the concentration of methotrexate and numbers of fibroblasts from patients with scleroderma and normal controls. A positive correlation was found between GAG synthesis in each cell, as measured by $\left[{ }^{3} \mathrm{H}\right]$ glucosamine and $\left[{ }^{35} \mathrm{~S}\right]$ sulphate incorporation, and increasing methotrexate concentrations in fibroblasts from patients with scleroderma and normal controls.

Conclusions-These data indicate increased GAG synthesis in scleroderma and normal fibroblasts with increasing concentrations of methotrexate. Therefore the reported beneficial effect of methotrexate on skin fibrosis in scleroderma is most probably not the result of direct inhibition of GAG synthesis by fibroblasts.
\end{abstract}

Department of

Rheumatology,

University Hospital

and University of

Nijmegen, Nijmegen,

The Netherlands

F H J van den Hoogen

$P M$ van der Kraan

A M T Boerbooms

W B van den Berg

L B A van de Putte

Department of Medica Statistics, University

Hospital and

University of

Nijmegen, Nijmegen,

The Netherlands

H J J van Lier

Correspondence to:

Dr Frank H J van den Hoogen,

Department of

Rheumatology, University

Hospital, PO Box 9101

$6500 \mathrm{HB}$ Nijmegen, The

Netherlands.

Accepted for publication

21 June 1993
(Ann Rheum Dis 1993; 52: 758-761)

Systemic sclerosis is characterised by proliferative vascular lesions, chronic inflammatory infiltrations, and an excessive accumulation of connective tissue in many organs. Abnormalities in the functioning of the fibroblasts are considered to be responsible for progressive fibrosis in systemic sclerosis. Tissue cultures of dermal fibroblasts from patients with systemic sclerosis have been found to synthesise collagen at an increased rate $^{12}$ and to accumulate up to five times more glycosaminoglycans (GAGs) than normal skin fibroblasts. ${ }^{34}$ There is evidence to suggest that most of the increase in GAG synthesis is in the hyaluronic acid fraction. ${ }^{34}$

Methotrexate in low doses is efficacious in treating several connective tissue diseases, such as rheumatoid arthritis (RA), ${ }^{56}$ dermato- myositis, and polymyositis. ${ }^{7}$ Low doses of methotrexate have also been reported to reduce skin thickening in patients with systemic sclerosis. ${ }^{89}$ The mechanisms through which low doses of methotrexate affect the inflammatory process in rheumatic disease are as yet unknown. Most studies of the immune system in patients with RA who have been treated with low doses of methotrexate show only marginal effects on the humoral and cellular immune responses. The rapid clinical responses to treatment and equally rapid flare up upon discontinuation suggest an antiinflammatory effect. ${ }^{10}$ One study suggests that one of the roles of methotrexate in RA may be to inhibit interleukin 1 mediated proliferation of synovial fibroblasts. ${ }^{11}$

This study was undertaken to determine the effects of increasing concentrations of methotrexate on the proliferation of cultured scleroderma skin fibroblasts and their production of GAGs.

\section{Patients and methods}

PATIENTS WITH SCLERODERMA AND NORMAL VOLUNTEERS

The nine patients fulfilled the American Rheumatism Association's preliminary criteria for the diagnosis of systemic sclerosis. ${ }^{12}$ The mean (SD) age of the patients was $49.4(8.4)$ years, with a range of 38-63 years. Seven patients were women and two were men. Five had diffuse systemic sclerosis - that is, with the skin proximal to the elbows affected-and four had limited systemic sclerosis - that is, with the skin distal to the elbows affected. Disease duration, estimated from the first signs of skin thickening, varied from six months to seven years, the median being 48 months. In six patients antibodies to topoisomerase I could be detected; in two others anticentromere antibodies were found. None of the patients had previously received any drug treatment known to influence connective tissue metabolism. The control group consisted of nine normal volunteers, five men and four women, with a mean (SD) age of $46.4(11 \cdot 7)$ years (range 29-67 years)

FIBROBLAST EXPLANT CULTURES

Full thickness skin biopsy samples were taken with a $4 \mathrm{~mm}$ punch from each patient at a site of dermal thickening on the dorsum of the left forearm; from each donor a biopsy sample was taken from either the dorsal forearm or the 
upper arm. Each biopsy specimen was minced and placed in a $25 \mathrm{~cm}^{2}$ plastic tissue culture flask (Costar, Cambridge, MA, USA), to which $4 \mathrm{ml}$ nutrient medium was added. The latter consisted of Dulbecco's modified Eagle's medium (DMEM; Flow Laboratories, Irvine, Strathclyde, UK) that contained $0.002 \mathrm{M}$ glutamine and 0.02 M HEPES buffer and had been supplemented with $15 \%$ fetal calf serum and gentamycin $(40 \mu \mathrm{g} / \mathrm{ml}$; Schering, Kenilworth, NJ, USA). In addition to HEPES buffer the medium $\mathrm{pH}$ was controlled by sodium hydrogencarbonate.

Each culture was maintained at $37^{\circ} \mathrm{C}$ in a humidified atmosphere of a $\mathrm{CO}_{2}$ incubator containing $5 \% \mathrm{CO}_{2}$ in air. The medium was completely replaced one to three times a week, depending on the change in colour. Once fibroblasts had grown from an explant culture, the old medium was discarded and fibroblasts were dispersed with $1 \mathrm{ml} \mathrm{0.05 \%}$ trypsin (Sigma Chemical, St Louis, MO, USA) for 15 minutes at $37^{\circ} \mathrm{C}$. The cells were left in the same flask and $4 \mathrm{ml}$ nutrient medium was added. When they reached confluence, the fibroblasts were trypsinised again and divided equally between two $25 \mathrm{~cm}^{2}$ tissue culture flasks to each of which $4 \mathrm{ml}$ nutrient medium was added. When these cell layers had become confluent, they were trypsinised once more and the dispersed fibroblasts of each subculture were divided equally among 20 wells of one of two identical 24 well tissue culture plates (Costar). A $500 \mu$ l volume of nutrient medium was added to each of these 40 wells. After 24 hours this was completely replaced with $500 \mu \mathrm{l}$ fresh medium containing methotrexate at a concentration of $0,10^{-8}, 10^{-6}, 10^{-4}$ or $10^{-2} \mathrm{~mol} / \mathrm{l}$. Each of the five concentrations was assigned to four wells on each plate. These cultures were then further incubated under the conditions described earlier. All subsequent determinations were performed on the contents of the quadruplicate wells.

MEASUREMENT OF GAG SYNTHESIS

The fibroblast cultures of one tissue culture plate were labelled with $185 \mathrm{kBq}\left[{ }^{3} \mathrm{H}\right]$ glucosamine (NEN Products, Dupont, Boston, MA,

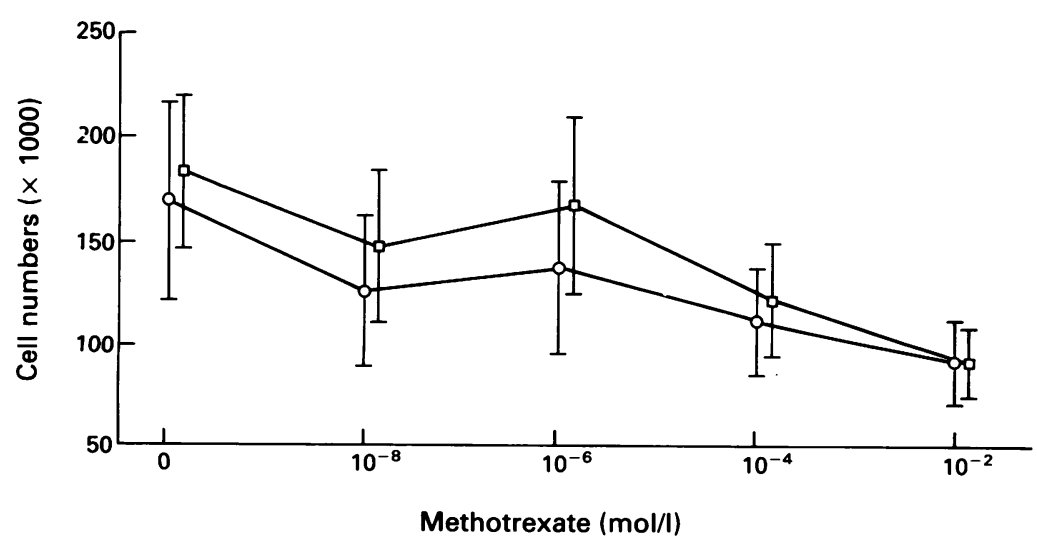

Figure 1 Effect of methotrexate on fibroblast proliferation. Methotrexate causes a dose dependent decrease of scleroderma $(p<0 \cdot 001)$ and normal $(p<0 \cdot 001)$ fibroblast numbers. No differences were observed between the numbers of scleroderma and normal fibroblasts. $-{ }_{-}$, scleroderma; $-\square-$, normal. Bars indicate SEM.
USA) and $185 \mathrm{kBq}\left[{ }^{35}\right.$ S] sulphate (NEN Products) in each well, three days after the methotrexate had been added. The next day, all medium was decanted from each well and supplemented with $200 \mu \mathrm{l}$ papain $(1 \mathrm{mg} / \mathrm{ml}$; Sigma Chemical). The fibroblasts were trypsinised $(200 \mu \mathrm{l} /$ well $)$ and mixed with 100 $\mu \mathrm{l}$ papain $(5 \mathrm{mg} / \mathrm{ml})$. The cells and decanted media were incubated at $60^{\circ} \mathrm{C}$ for 24 hours. A $0.2 \%$ solution of cetylpyridinium chloride (CPC) was then added, $400 \mu l$ to the fibroblasts and $600 \mu \mathrm{l}$ to the medium, to obtain a final concentration of $0 \cdot 1 \%$ in all samples. These were incubated at $37^{\circ} \mathrm{C}$ for one hour to allow the GAG-CPC complex to precipitate.

The resulting pellets were centrifuged and washed, the cell fraction twice and the medium fraction three times, with $0.05 \%$ CPC to remove any remaining glycopeptides and unincorporated precursors. Each pellet was then supplemented with $0.5 \mathrm{ml}$ Luma solve (Lumac.LSC, Olen, Belgium) and, after an incubation of 10 minutes at $60^{\circ} \mathrm{C}, 10 \mathrm{ml}$ Lipoluma (Lumac.LSC) was added. The $\left[{ }^{3} \mathrm{H}\right]$ glucosamine and $\left[{ }^{35} \mathrm{~S}\right]$ sulphate content of the pellets was determined in a liquid scintillation counter (LKB, Sweden) and appropriate corrections for the $\left[{ }^{3} \mathrm{H}\right]-\left[{ }^{35} \mathrm{~S}\right]$ overlap were made. As these experiments were not performed simultaneously, the specific activities of the isotopes had been determined before labelling. The results were corrected for the decrease in radioactivity that occurred during the course of the study to obtain results for the levels of incorporation that could be compared.

\section{FIBROBLAST COUNT}

The second tissue culture plate was used for counting cells. Four days after methotrexate had been added to the cultures, the media were decanted. The cells were washed twice with $400 \mu \mathrm{l}$ phosphate buffered saline per well. Following trypsinisation $(200 \mu \mathrm{l} /$ well $)$, the cells of each well were counted with a Bürker haemocytometer. The $\left[{ }^{3} \mathrm{H}\right]$ and $\left[{ }^{35} \mathrm{~S}\right]$ counts/ minute (cpm) of each of the quadruplicate cultures were divided by the corresponding number of cells to obtain the GAG synthesis for each fibroblast, to eliminate the effect of methotrexate on cell proliferation.

STATISTICAL ANALYSIS

For all of the variables the means were calculated for quadruplicate cultures. Comparisons between groups were performed with the two tailed Wilcoxon test. Spearman rank correlation coefficients were calculated, and Fisher's Z method applied. p Values of 0.01 or less were considered significant.

\section{Results}

FIBROBLAST PROLIFERATION

As shown in fig 1, the mean number of cells was significantly inversely related to the concentration of methotrexate to which they had been exposed. The mean number of sclero- 

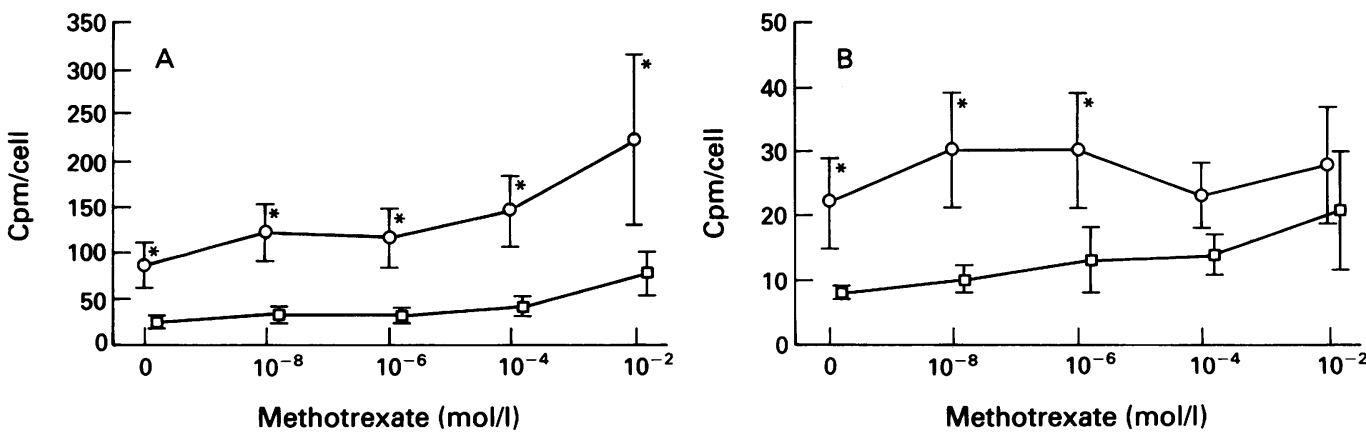

Figure 2 Mean total $\left[{ }^{3} \mathrm{H}\right](A)$ and $\left[{ }^{35} \mathrm{~S}\right](B)$ counts per minute (cpm) per cell of scleroderma and normal fibroblasts. Each value represents the mean and SEM of quadruplicate cultures. A positive correlation was found between the concentration of methotrexate and both total $\left[{ }^{3} \mathrm{H}\right] \mathrm{cpm}$ of scleroderma $(p<0.001)$ and normal $(p<0.001)$ fibroblasts and total $\left.{ }^{35} \mathrm{~S}\right] \mathrm{cpm}$ of scleroderma $(p<0.001)$ and normal fibroblasts $(p<0.001)$. An asterisk indicates a significant difference $(p<0 \cdot 01)$. $-0-$, scleroderma; $-\square$, normal. Bars indicate SEM.

derma fibroblasts in each well was lower than that of control fibroblasts for all concentrations of methotrexate except $1 \times 10^{-2} \mathrm{~mol} / \mathrm{l}$, but the differences were not significant.

\section{GAG SYNTHESIS}

There was a positive correlation between the total amount of $\left[{ }^{3} \mathrm{H}\right]$ (mean $\left[{ }^{3} \mathrm{H}\right]$ count rate in cells plus mean $\left[{ }^{3} \mathrm{H}\right]$ count rate in media of quadruplicate cultures) and the corresponding concentration of methotrexate for scleroderma and normal fibroblasts (fig 2A). Likewise, the total $\left[{ }^{35} \mathrm{~S}\right]$ count rate was positively correlated with the corresponding concentration of methotrexate (fig 2B).

As the extent of skin disease and disease duration in systemic sclerosis may influence GAG synthesis, we compared the results for fibroblast cultures derived from specimens from five patients with diffuse skin disease with those for cultures derived from specimens from four patients with limited skin disease. For each concentration of methotrexate the total $\left[{ }^{3} \mathrm{H}\right]$ and $\left[{ }^{35} \mathrm{~S}\right]$ count rates were higher for diffuse disease fibroblasts than for limited disease fibroblasts $(p<0 \cdot 01)$. There was no difference between the total $\left[{ }^{3} \mathrm{H}\right]$ or $\left[{ }^{35} \mathrm{~S}\right]$ count rate of limited disease fibroblasts and those of normal fibroblasts.

The results for fibroblast cultures derived from specimens from five patients with a disease duration of more than three years were compared with those derived from specimens from four patients with a disease duration of less than three years. The total $\left[{ }^{3} \mathrm{H}\right]$ and $\left[{ }^{35} \mathrm{~S}\right]$ count rates were higher for patients with long term disease than for those with short term

Mean (SD) percentages of $\left[{ }^{3} \mathrm{H}\right]$ glucosamine and $\left[{ }^{35} \mathrm{~S}\right]$ sulphate count rates secreted into the medium by dermal fibroblasts of patients with scleroderma and control subjects. Differences between scleroderma and normal fibroblasts were not significant for any methotrexate concentration

\begin{tabular}{llllll}
\hline \multicolumn{5}{l}{ Methotrexate concentration (moll) } \\
\cline { 2 - 6 } & 0 & $1 \times 10^{8}$ & $1 \times 10^{-6}$ & $1 \times 10^{-4}$ & $1 \times 10^{-2}$ \\
\hline$\left[{ }^{3} \mathrm{H}\right]$ medium & & & & & \\
$\quad$ Scleroderma fibroblasts & $80(8)$ & $76(8)$ & $75(7)$ & $81(7)$ & $83(5)$ \\
$\quad$ Normal fibroblasts & $75(6)$ & $77(8)$ & $74(7)$ & $77(9)$ & $80(7)$ \\
& & & & & \\
{$\left[\begin{array}{l}\left.{ }^{35} \text { S }\right] \text { medium } \\
\text { Scleroderma fibroblasts }\end{array}\right.$} & $65(11)$ & $62(7)$ & $59(8)$ & $60(11)$ & $62(11)$ \\
$\quad$ Normal fibroblasts & $57(9)$ & $58(12)$ & $56(12)$ & $57(13)$ & $62(15)$ \\
\hline
\end{tabular}

disease, but the differences were significant for a methotrexate concentration of $1 \times 10^{-4} \mathrm{~mol} / 1$ only. Values of total $\left[{ }^{3} \mathrm{H}\right]$ and $\left[{ }^{35} \mathrm{~S}\right]$ count rates of normal fibroblasts were between the values for fibroblasts of patients with long term and short term disease and were not significantly different from either of these.

More than half the newly synthesised GAGs were secreted into the media (table). Scleroderma and normal fibroblasts secreted a higher percentage of $\left[{ }^{3} \mathrm{H}\right]$ glucosamine labelled GAGs into the media than $\left[{ }^{35} \mathrm{~S}\right]$ sulphate labelled GAGs $(p<0 \cdot 001)$.

\section{Discussion}

Methotrexate has been reported to be efficacious in the treatment of systemic sclerosis and particularly in treating the affected skin. ${ }^{89}$ If the beneficial effect of methotrexate is a result of it acting directly on the fibroblasts, this might be either by inhibiting their proliferation or by decreasing the production of extracellular matrix. Methotrexate, an antagonist of folate dependent enzymes, interferes with the de novo pyrimidine and purine synthesis of RNA and DNA and, hence, blocks cell proliferation. The inverse relation between the number of cells and methotrexate concentrations in scleroderma and normal fibroblasts was therefore to be expected. The proliferation rate was approximately the same in scleroderma and normal fibroblasts.

The total $\left[{ }^{3} \mathrm{H}\right]$ and $\left[{ }^{35} \mathrm{~S}\right]$ count rates were positively correlated with the concentration of methotrexate for scleroderma and normal fibroblasts. As total $\left[{ }^{3} \mathrm{H}\right]$ and $\left[{ }^{35} \mathrm{~S}\right]$ count rates reflect the incorporation of $\left[{ }^{3} \mathrm{H}\right]$ glucosamine and $\left[{ }^{35} \mathrm{~S}\right]$ sulphate into $\mathrm{GAGs}$, these results show that, in scleroderma and normal fibroblast cultures, GAG synthesis increases with increasing concentrations of methotrexate.

Production of GAGs by scleroderma fibroblasts as measured iy the total $\left[{ }^{3} \mathrm{H}\right]$ count rate was significantly greater than that by normal fibroblasts for all concentrations of methotrexate. The production of GAGs as measured by the total $\left[{ }^{35} \mathrm{~S}\right]$ count rate was significantly greater in scleroderma fibroblasts in the absence of methotrexate and at concentrations of $1 \times 10^{-8}$ and $1 \times 10^{-6} \mathrm{~mol} / \mathrm{l}$. As $\left[{ }^{3} \mathrm{H}\right]$ glucos- 
amine is incorporated into all GAGs, and $\left[{ }^{35} \mathrm{~S}\right]$ sulphate into sulphate containing GAGs only, thus leaving hyaluronic acid unlabelled by $\left[{ }^{35} \mathrm{~S}\right]$ sulphate, this indicates an enhanced accumulation of hyaluronic acid in scleroderma fibroblasts at higher methotrexate concentrations. The possibility that undersulphated GAGs are produced cannot be totally excluded, however.

As diffuse skin disease was associated with a greater production of GAGs than limited skin disease, which was associated with a level of production similar to that of normal fibroblasts, the increased production of GAGs by scleroderma fibroblasts must be attributed primarily to those derived from the biopsy specimens from patients with diffuse skin disease.

Systemic sclerosis is believed to be at its most active during the first three years. We had, therefore, expected a greater production of GAGs to be associated with short disease duration than with long disease duration. We found, however, a greater production of GAGs in fibroblast cultures of patients with long term disease. The difference was not significant, perhaps because the number of patients was too small.

The concentration of methotrexate had no effect on the percentages of newly synthesised $\left[{ }^{35} \mathrm{~S}\right]$ sulphate or $\left[{ }^{3} \mathrm{H}\right]$ glucosamine labelled GAGs that were secreted into the media by the scleroderma fibroblasts, which were comparable with those found by Bashey et al. ${ }^{4}$ This shows that methotrexate does not cause a shift in the distribution of GAGs between the cells and medium.

Considering the mechanisms through which methotrexate is known to act, we had expected GAG synthesis to decrease as the concentration of methotrexate increased. The results show, however, that GAG production by cultured fibroblasts, whether scleroderma or normal, actually increases with increasing methotrexate concentrations. There are several possible explanations for this. First, the synthesis of extracellular matrix may be suppressed through contact inhibition. ${ }^{13}$ As shown in fig 1 , methotrexate reduces the number, and therefore the density, of fibroblasts as its concentration increases, thus creating a population in which less contact inhibition can occur. This could lead to more GAG synthesis for each fibroblast. Second, methotrexate might have been responsible for selecting those fibroblasts with high rates of GAG synthesis. It has been established that normal and scleroderma fibroblasts are heterogeneous with respect to their synthetic and proliferative capabilities and that systemic sclerosis fibroblasts are somehow selected so that high producers of connective tissue are favoured at the expense of low producers, which they replace. ${ }^{14}$ Third, the increase in
GAG synthesis may be due primarily to decreased degradation as a result of the interference by methotrexate with the synthesis of GAG degrading enzymes, rather than to increased production. Finally, by blocking DNA synthesis and thus cell proliferation, methotrexate may promote a fibroblast differentiation that is accompanied by protein synthesis. Methotrexate has been reported to induce the differentiation of cultured human keratinocytes, resulting in an increase in protein synthesis by a factor of $2-2 \cdot 3 .{ }^{15}$

The results obtained in this study are inconsistent with the reported beneficial effect of low doses of methotrexate of the skin in systemic sclerosis. Therefore any beneficial effect of methotrexate on skin thickening in systemic sclerosis is most probably not the result of direct inhibition of GAG production by fibroblasts, but is more likely to be based on modulation of the (immuno)inflammatory system.

This work was supported by the Dutch League Against Rheumatism. The authors thank E Vitters for her skilful technical assistance.

1 LeRoy E C. Increased collagen synthesis by scleroderma skin fibroblasts in vitro. A possible defect in the regulation or activation of the scleroderma fibroblast. $\mathcal{F}$ Clin Invest 1974; 54: 880-9.

2 Uitto J, Bauer E A, Eisen A Z. Scleroderma. Increased biosynthesis of triple-helical type I and type III procollagens associated with unaltered expression of collagenase by skin fibroblasts in culture. $\mathcal{f}$ Clin Invest 1979; 64: $921-30$

3 Cabral A, Castor C W. Connective tissue activation XXVII. The behavior of skin fibroblasts from patients with scleroderma. Arthritis Rheum 1983; 26: 1362-9.

4 Bashey R I, Millan A, Jimenez S A. Increased biosynthesis of glycosaminoglycans by scleroderma fibroblasts in culture. Arthritis Rheum 1984; 27: 1040-5.

5 Weinblatt M E, Coblyn J S, Fox D A, et al. Efficacy of lowdose methotrexate in rheumatoid arthritis. $N$ Engl $\mathcal{F ~ M e d ~}$ 1985; 312: 818-22.

6 Jeurissen M E C, Boerbooms A M Th, van de Putte L B $\mathrm{A}$, et al. Methotrexate versus azathioprine in the treatment of rheumatoid arthritis. Arthritis Rheum 1991; 34: 961-71.

7 Metzger A L, Bohan A, Goldberg L S, Bluestone R, Pearson C M. Polymyositis and dermatomyositis: combined methotrexate and corticosteroid therapy. Ann Intern Med 1974; 81: 182-9.

8 van den Hoogen F H J, Boerbooms A M T, van de Putte L B A, Rasker J J, van Venrooij W J. Low dose methotrexate treatment in systemic sclerosis. f Rheumatol methotrexate treatm

9 Bode B Y, Yocum D E, Gall E P, et al. Methotrexate (MTX) in scleroderma: experience in ten patients [abstract]. Arthritis Rheum 1990; 33(suppl 9): S66.

10 Segal R, Yaron M, Tartakovsky B. Methotrexate: mechanism of action in rheumatoid arthritis. Semin Arthritis Rheum 1990; 20: 190-9.

11 Yaron M, Yaron I, Meyer F A. Methotrexate inhibits interleukin-1 mediated synovial fibroblast proliferation [abstract]. Arthritis Rheum 1991; 34(suppl 9): S92.

12 Subcommittee for Scleroderma Criteria of the American Rheumatism Association Diagnostic and Therapeutic Criteria Committee. Preliminary criteria for the classification of systemic sclerosis (scleroderma). Arthritis Rheum 1980; 23: 581-90.

13 Lackie J M. Cell-cell interactions. In: Lackie J M, ed. Cell movement and cell behaviour. London: Allen and Unwin, 1986: 253-76.

14 White Needleman B, Ordonez J V, Taramelli D, Alms W, Gayer K, Choi J. In vitro identification of a subpopulation of fibroblasts that produces high levels of collagen in scleroderma patients. Arthritis Rheum 1990; 33: 842-52.

15 Schwartz P M, Barnett S K, Atillasoy E S, Milstone L M. Methotrexate induces differentiation of human keratinocytes. Proc Natl Acad Sci USA 1992; 89: 594-8. 